\title{
Impact de l'érosion sur l'envasement des barrages, la recharge des nappes phréatiques côtières et les intrusions marines dans la zone semi-aride méditerranéenne : cas du barrage de Boukourdane (Algérie)
}

\author{
Nassima Tadrist ${ }^{(1,2)}$, Olivier Debauche ${ }^{(3,4)}$, Boualem Remini ${ }^{(2)}$, Dimitri Xanthoulis ${ }^{(1)}$, \\ Aurore Degré (1) \\ (1) Université de Liège - Gembloux Agro-Bio Tech. Biosystems Engineering (BIOSE). Axe Échanges Eau-Sol-Plante. \\ Passage des Déportés, 2. BE-5030 Gembloux (Belgique).E-mail : nassima.tadrist@gmail.com \\ (2) Université Saâd Dahlab de Blida. Département des Sciences de l'Eau et de l'Environnement. Faculté de Technologie. \\ Route de Soumâa. BP 270. 09000 Blida (Algérie). \\ (3) Epuvaleau ASBL. Passage des Déportés, 2. BE-5030 Gembloux (Belgique). \\ (4) Université de Mons. Faculté Polytechnique. Service d'Informatique. Rue de Houdain, 9. BE-7000 Mons (Belgique).
}

Reçu le 1 janvier 2015, accepté le 31 aout 2016.

Description du sujet. L'érosion hydrique est une problématique particulièrement importante, notamment dans la zone méditerranéenne et semi-aride. Cette zone est caractérisée par des régimes pluviométriques irréguliers qui ont une influence considérable sur les pertes en sol. Dans les zones montagneuses, les phénomènes d'érosion hydrique sont accentués par les fortes pentes et la couverture réduite du sol. Les barrages alimentés par ces zones subissent un envasement lié à une érosion importante. L'envasement de ces barrages limite drastiquement leur capacité et donc leur durée d'exploitation. Lorsqu'ils sont utilisés pour la recharge de nappes phréatiques, les lâchers entrainent la remise en suspension des particules fines qui s'accumulent au niveau des zones de recharge et réduisent la capacité d'infiltration par colmatage de la porosité du sol, ce qui conduit à un abattement important des niveaux piézométriques. De plus, les nappes phréatiques situées dans la zone côtière sont sujettes à des pompages intensifs. Ces deux phénomènes ont pour conséquence de rendre les nappes particulièrement sensibles aux intrusions marines.

Objectifs. Cet article vise, d'une part, à quantifier le taux d'envasement dans le cas du barrage de Boukourdane (Nord de l'Algérie), qui est utilisé pour la recharge des nappes phréatiques et à montrer, d'autre part, la diminution de la perméabilité au niveau du champ captant avec la gestion actuelle des lâchers.

Méthode. Les débits solides ontété mis relation avec les débits liquides et la concentration en sédiments prélevés ponctuellement dans l'oued. La relation établie a permis d'évaluer les apports solides dans le barrage de Boukourdane. L'impact des lâchers sur la recharge du champ captant a été évalué par regroupement des puits et forages du champ captant à l'aide de l'heuristique k-moyenne. Des régressions ont ensuite été appliquées sur les variations piézométriques de chacun des groupes.

Résultats. Le taux d'érosion spécifique des sols, aussi appelé taux de dégradation spécifique des sols, a été évalué à $366 \mathrm{t} \cdot \mathrm{km}^{-2}$. $\mathrm{an}^{-1}$ pour la période de 1993 à 2005. L'augmentation de la fréquence des pluies exceptionnelles se traduit par une augmentation du taux d'érosion des sols à $446 \mathrm{t} \cdot \mathrm{km}^{-2} \cdot \mathrm{an}^{-1}(+22 \%)$ pour la période de 1993 à 2013. Ces valeurs correspondent à des volumes de vase accumulés au droit du barrage respectivement de $8 \cdot 10^{4} \mathrm{~m}^{3} \cdot \mathrm{an}^{-1}$ et $11 \cdot 10^{4} \mathrm{~m}^{3} \cdot \mathrm{an}^{-1}$.

Conclusions. Une modification de la gestion des lâchers actuelle a un impact positif sur la restauration partielle de la conductivité hydraulique et de facto, sur la lutte contre les intrusions marines.

Mots-clés. Recharge de la nappe, barrage, sédimentation, érosion hydrique, Algérie.

Impact of erosion on the silting of dams, on the recharging groundwater and on coastal marine intrusion in the Mediterranean semi-arid area: Case of Boukourdane dam (Algeria)

Description of the subject. Water erosion is a particularly important issue, especially in the Mediterranean and semi-arid zone. This zone is characterized by irregular rainfall patterns, which have a considerable influence on soil loss. In mountainous areas, water erosion phenomena are accentuated by steep slopes and low ground cover. The dams draining these areas undergo 
siltation linked to significant erosion. The silting of the dams drastically limits their capacity and thus their operating life. When used for recharging aquifers, the resuspension of fine particles may accumulate in the recharge areas and reduce infiltration capacity by clogging soil porosity. This leads to a significant reduction of groundwater levels. Additionally, groundwater situated in the coastal zone is submitted to intensive pumping. These two phenomena make groundwater particularly sensitive to marine intrusions.

Objectives. This article aims, on the one hand to quantify the rate of siltation in the case of the Boukourdane dam (northern Algeria), which is used to recharge groundwater. On the other hand, the article also shows the reduction in permeability in the capturing field with the current management of releases.

Method. Solid rates were set in relation to the liquid flow and sediment concentration occasionally taken from the wadi. The relation established made it possible to evaluate the solid contributions to the dam of Boukourdane. The impact of the releases on recharging the well field was evaluated by grouping wells and the well field mushrooms using heuristic k-means. Regressions were applied to the piezometric variations of each group.

Results. This dam is fed by a catchment area of $156 \mathrm{~km}^{2}$. Sediment transport was estimated from empirical formulas. The specific degradation rate was estimated at $366 \mathrm{t} \cdot \mathrm{km}^{-2} \cdot \mathrm{year}^{-1}$ during the 1993 to 2005 period. The increase in the frequency of extreme rainfall resulted in an increase in the soil erosion rate to $446 \mathrm{t} \cdot \mathrm{km}^{-2} \cdot \mathrm{year}^{-1}$ during the 1993 to 2013 period. The volume of sediments accumulated in the dam reached $8 \cdot 10^{4} \mathrm{~m}^{3} \cdot \mathrm{year}^{-1}$ and $11 \cdot 10^{4} \mathrm{~m}^{3} \cdot \mathrm{year}^{-1}$ respectively during these two periods.

Conclusions. A change in the management of current releases has a positive impact on the partial restoration of the hydraulic conductivity and de facto, on the fight against marine intrusion.

Keywords. Groundwater recharge, dams, sedimentation, water erosion, Algeria.

\section{INTRODUCTION}

Les zones méditerranéennes et semi-arides présentent des conditions naturelles rudes caractérisées par un climat très agressif en automne avec des précipitations très irrégulières pouvant présenter de fortes intensités dépassant les $45 \mathrm{~mm} \cdot \mathrm{h}^{-1}$ (Touaïbia, 2000).

Dans le sud du bassin méditerranéen, le climat semi-aride est un facteur prépondérant dans la gravité du phénomène d'érosion hydrique. En effet, le climat semi-aride est caractérisé par des périodes sèche et humide ainsi que de brusques variations spatio-temporelles des quantités de précipitation. L'évapotranspiration potentielle excède souvent les précipitations. Ces précipitations, dans les zones montagneuses, engendrent des écoulements torrentiels (Yahiaoui, 2012). Les écoulements résultant de ce régime torrentiel arrachent les particules du sol, les transportent dans les oueds et sédimentent derrière les barrages. L'évolution climatique de ces dernières années a accentué les périodes de sècheresse qui ont touché l'ensemble des zones méditerranéennes (Medejerab et al., 2011 ; Szczypta, 2012). Les périodes de sècheresse ont eu comme conséquence de compliquer l'approvisionnement en eau potable effectué à partir des réserves stockées au niveau des barrages. L'envasement excessif de la plupart des grands barrages algériens a considérablement limité leur volume utile et donc leur capacité réelle de stockage. Bien que le transport solide par l'eau de pluie et de ruissellement soit un processus naturel, il est fortement accentué par les activités anthropiques: surpâturage, culture sans restitution, divers méfaits comme l'incendie, dans les années 1990, de la végétation ou le déboisement fragilisant les sols qui se retrouvent parfois dégradés et laissent apparaitre la roche mère. Il menace les ressources en sol et en eau non seulement par l'érosion «on site » et la perte de terres mais aussi par la sédimentation «off site ».

L'estimation du taux de sédimentation dans les barrages et leur durée de vie nécessitent une connaissance des apports solides provenant du bassin versant. La connaissance des exportations de sédiments permet de sélectionner les bassins versants qui seront prioritairement investigués et éventuellement aménagés en vue de les réduire drastiquement ou de mieux les gérer. Une analyse plus fine, recourant à une modélisation basée sur le type de sol, les pentes et le couvert végétal permettra par la suite de cibler les zones les plus sensibles à aménager au sein de ces bassins versants.

La problématique du transport solide et l'ampleur du phénomène d'envasement dans les barrages ont suscité l'intérêt de nombreux chercheurs depuis les années 1950. De nombreux modèles explicatifs du transport solide à partir de paramètres comme le débit liquide, la lame d'eau ruisselée, la superficie et la pente moyenne du bassin versant, l'humidité du sol, la pluie ont été élaborés par différents auteurs pour le Maghreb. Le tableau 1 reprend les principales contributions en matière d'estimation du taux d'érosion spécifique des sols $\left(t \cdot \mathrm{km}^{-2} \cdot \mathrm{an}^{-1}\right)$, obtenu en rapportant le flux sédimentaire à l'aire du bassin versant. En d'autres termes, l'érosion spécifique est le taux d'érosion moyen sur le bassin versant.

Le taux d'érosion spécifique des sols dans le sud du bassin méditerranéen varie entre 1000 et $5000 \mathrm{t}$. $\mathrm{km}^{-2} \cdot \mathrm{an}^{-1}$ (Walling, 1984). Toutefois, plusieurs auteurs ont montré que ces valeurs pouvaient être dépassées localement. On note les $7200 \mathrm{t} \cdot \mathrm{km}^{-2} \cdot \mathrm{an}^{-1}$ pour l'oued Agrioun alimentant le barrage d'Ighil Emda en Algérie 
Tableau 1. Valeurs des taux d'érosion spécifiques des sols des bassins versants du Maghreb - Values of specific erosion rates of the soils of Maghreb watersheds.

\begin{tabular}{lcl}
\hline Bassin versant (région) & $\begin{array}{c}\text { Érosion spécifique } \\
\text { des sols }\left(\mathrm{t} \cdot \mathrm{km}^{-2} \cdot \mathrm{an}^{-1}\right)\end{array}$ & Référence \\
\hline Abd (Algérie) & 136 & Achite et al., 2007 \\
\hline Algérie orientale & 145 & Bourouba, 1998 \\
\hline Bellah (Algérie) & 610 & Elahcene et al., 2013 \\
\hline Boukadir (Algérie) & 446 & Présente étude \\
\hline Ebda (Algérie) & 1875 & Meddi, 1999 \\
\hline El-Hachem (Algérie) & 318 & Tadrist, 2004 \\
\hline Kebir bassin (Algérie) & 572 & Khanchoul et al., 2012 \\
\hline La haute Tafna (Algérie) & 1120 & Megnounif et al., 2003 \\
\hline La Tafna (Algérie) & 150 & Sogreah, 1967 \\
\hline L'Isser (Lakhdaria) & 2300 & Bourouba, 1997 \\
\hline Maghreb & 265 à 2569 & Heusch et al., 1971 \\
\hline Maghreb & 1000 à 5000 & Walling, 1984 \\
\hline Maghreb & 397 & Probst et al., 1992 \\
\hline Mazafran (Côtier algérois) & 1610 & Bourouba, 1996 \\
\hline Mina (Algérie) & 187 & Achite et al., 2005 \\
\hline Mouilab (Algérie) & 126 & Terfous et al., 2001 \\
\hline Nekor (Maroc) & 5900 & Lahlou, 1988 \\
\hline Oued Agrioun (Algérie) & 7200 & Probst et al., 1992 \\
\hline Saf Saf (Algérie) & 461 & Khanchoul et al., 2007 \\
\hline Sebdou (Algérie) & 1330 & Ghenim et al., 2008 \\
\hline Sebdou (Algérie) & 937 & Bouanani, 2004 \\
\hline Sebdou (Algérie) & 343 & Megnounif et al., 2013 \\
\hline Sikkak (Algérie) & 170 & Bouanani, 2004 \\
\hline Souagui II (Algérie) & 1268 & Keddar, 2011 \\
\hline Tebaga (Tunisie centrale) & 336 & Bergaoui et al., 1998 \\
\hline & & \\
\hline
\end{tabular}

destination des villes environnantes, il est également utilisé pour la recharge de la nappe phréatique du champ captant situé en aval.

Les objectifs de cette étude sont de quantifier le transport des matières en suspension qui s'accumulent dans le barrage, mais également de montrer l'impact de ces matières en suspensions sur la conductivité hydraulique du champ captant, situé en aval du barrage, en utilisant l'analyse des variations piézométriques avant et après lâchers de barrage et le regroupement des puits et forages à l'aide de l'algorithme k-moyennes.

\section{MATÉRIEL ET MÉTHODES}

\subsection{Caractéristiques de la zone d'étude}

La région étudiée, située dans la wilaya (division administrative) de Tipasa au nord de l'Algérie, fait partie de l'Atlas tellien-littoral (Figure 1). L'impluvium du barrage de Boukourdane couvre une superficie de $156 \mathrm{~km}^{2}$ pour un périmètre de $63 \mathrm{~km}$. Il est situé entre les longitudes $2^{\circ} 7^{\prime} 40^{\prime \prime}$ et $2^{\circ} 20^{\prime} 21^{\prime \prime}$ Est et les latitudes $36^{\circ} 32^{\prime} 60^{\prime \prime}$ et $36^{\circ} 24^{\prime} 43^{\prime \prime}$ Nord. Il est alimenté par l'oued El Hachem. Comme le montre la figure $\mathbf{1}$, ce dernier prend naissance suite à la confluence des quatre principaux affluents: l'oued Boukadir en provenance de l'Ouest, l'oued Tegdza en provenance du Sud et les oueds Nachef et Fedjena venant

(Probst et al., 1992) et les $5900 \mathrm{t} \cdot \mathrm{km}^{-2} \cdot \mathrm{an}^{-1}$ enregistrés au niveau du bassin versant du Nekor qui alimente le barrage de Mohamed El Khettabi au Maroc (Lahlou, 1988). Parallèlement à ces fortes valeurs d'érosion spécifiques des sols, d'autres auteurs ont publié des estimations en dessous de cette fourchette. Bouanani (2004) a calculé pour l'oued Sikkak (Algérie) 170 t. $\mathrm{km}^{-2} \cdot \mathrm{an}^{-1}$ et Bergaoui et al. (1998) ont évalué à $336 \mathrm{t}$. $\mathrm{km}^{-2} \cdot \mathrm{an}^{-1} \mathrm{l}^{\prime}$ érosion spécifique des sols du bassin versant de Tebaga en Tunisie centrale.

Dans le cadre de cette étude, le choix s'est porté sur le barrage de Boukourdane en Algérie, car ce barrage illustre bien les phénomènes d'envasement que subissent tous les grands barrages situés dans le sud-ouest du bassin méditerranéen (Remini et al., 2005). Outre sa fonction de réservoir d'eau potable à de l'Est (Fareh, 2006).

L'altitude du bassin versant de l'oued El Hachem varie entre 100 et $1417 \mathrm{~m}$ avec une moyenne de $387 \mathrm{~m}$. La densité de drainage est de $3,10 \mathrm{~km} \cdot \mathrm{km}^{-2}$, la longueur de son talweg principal est de $34 \mathrm{~km}$.

Les pentes supérieures à $35 \%$ occupent une superficie de 78,68 $\mathrm{km}^{2}$ de l'amont du bassin versant, tandis que les pentes entre 6 et $35 \%$ occupent une superficie de $68,51 \mathrm{~km}^{2}$ des zones collinaires de la partie centrale du bassin versant. Les faibles pentes, de 0 à $6 \%$, se localisent principalement dans la vallée et occupent une superficie de $10,87 \mathrm{~km}^{2}$ dans la partie aval du bassin versant.

Les principaux types de sols identifiés dans le bassin versant de l'oued El Hachem sont de types Leptosol lithique, Régosol calcaire et Cambisol (WRB). 
Tadrist N., Debauche O., Remini B. et al.

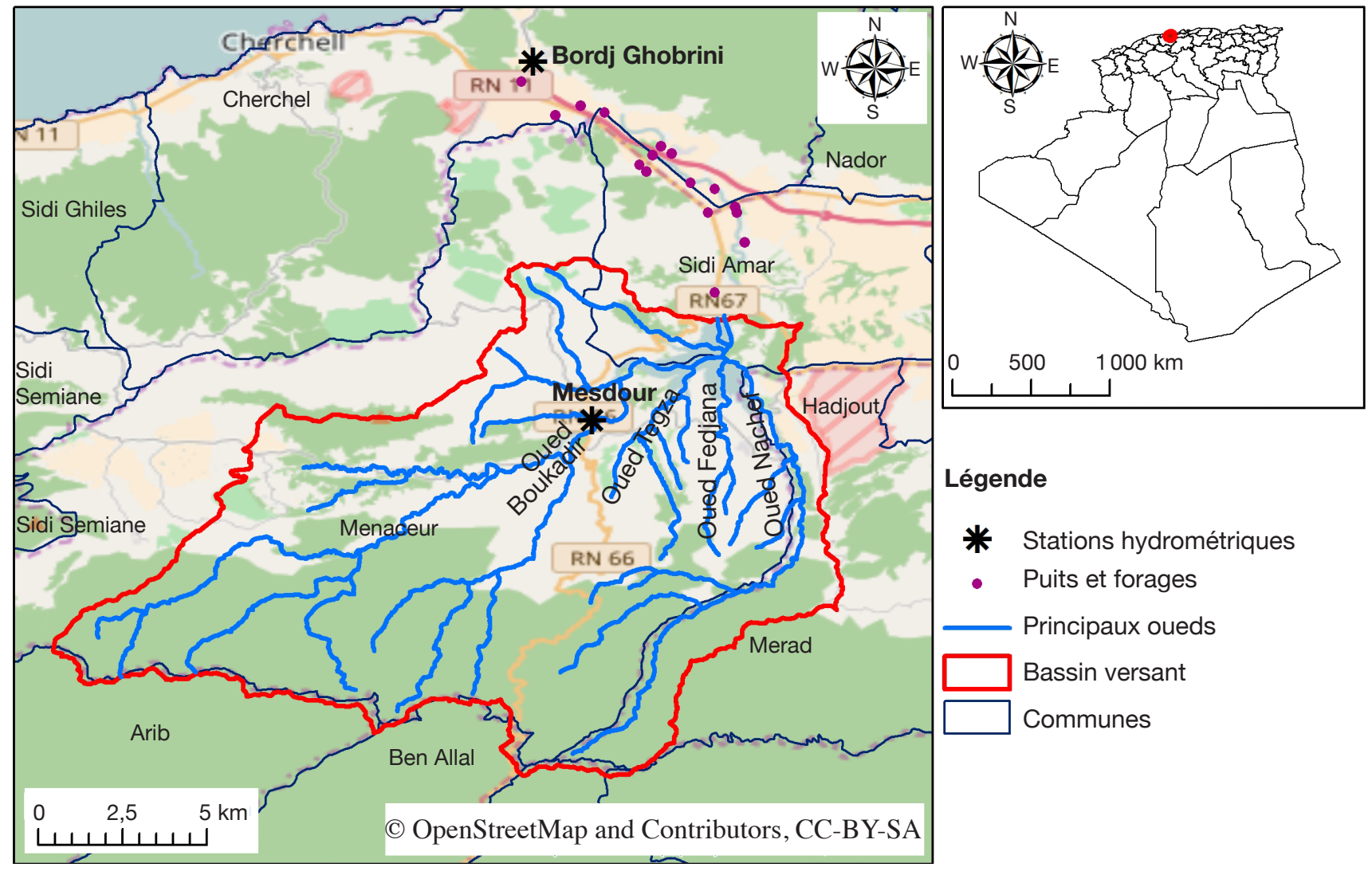

Figure 1. Localisation de la zone d'étude - Location of the study area.

Sources : GADM, Agence Nationale des Ressources Hydrauliques (ANRH).

Le diagramme des textures américain montre une prédominance des granulométries très fine et fine qui correspondent aux textures argileuse à limoneuse, sur l'ensemble des 70 profils réalisés sur la zone d'étude dont 16 ont fait l'objet d'analyses en laboratoire. Le taux d'argile varie de 12,6 à $46 \%$.

Les sols de type Leptosol lithique occupent les sommets des montagnes sur une superficie de 207 ha. Sur les glacis, les sols de type Calcisol sont caractérisés par la présence d'une croute calcaire à moins de $30 \mathrm{~cm}$ de profondeur. Les Calcisols occupent une superficie de 1977 ha. Ils sont présents dans la plaine et sont caractérisés par la présence d'un encroutement calcaire à différentes profondeurs. Les Régosols sont des sols caillouteux peu profonds et faiblement structurés. Ils sont présents près des sommets des montagnes, les zones de pentes fortes et occupent une surface de 3522 ha. Les Cambisols couvrent une surface de 6250 ha. Ils sont localisés sur les collines et basses collines. Ce sont des sols moyennement profonds assez riches en argile, mais présentant une structure faiblement évoluée surtout en profondeur, ainsi que des cailloux et colluvions.

Le tableau 2 reprend les principales caractéristiques des sols rencontrés sur le bassin versant.
Les différentes formations géologiques fréquemment rencontrées sur la zone d'étude sont des roches sédimentaires très érodables, à l'origine de la grande sensibilité des sols de la région vis-à-vis du processus d'érosion. Il s'agit là essentiellement de schistes, marnes tendres et schisteuses et de calcaires.

Le couvert végétal de la région de l'oued El Hachem est dominé par des forêts constituées de chêne liège (Quercus suber), de chêne vert (Quercus ilex), pins (Pinus sp.), cyprès (Cupressus sp.), genévriers (Juniperus sp.), peupliers (Populus sp.), de formations arbustives et de maquis. Dans les zones agricoles, on rencontre des terrains en friche ou labourés, des parcelles de céréales, de pomme de terre, du maraichage, de l'arboriculture fruitière, des oliviers et des vignes.

Le climat du bassin versant de l'oued El Hachem est de type semi-aride, c'est-à-dire humide et froid en hiver, chaud et sec en été.

Comme le montre la figure 2 , les mois les plus pluvieux sont novembre $(101 \mathrm{~mm})$ et décembre (105 mm).

Le bassin versant reçoit annuellement une pluviométrie située entre $300 \mathrm{~mm}$ et $750 \mathrm{~mm}$ avec une moyenne de $523 \mathrm{~mm}$ calculée sur la période 
Tableau 2. Principales caractéristiques d'un profil type de chaque sol présent dans le bassin versant - Main features of a typical profile of each soil present in the watershed.

\begin{tabular}{lccccc}
\hline Type de sol & $\begin{array}{l}\text { Teneur en argile } \\
(\%)\end{array}$ & $\begin{array}{l}\text { Teneur en limon } \\
(\%)\end{array}$ & $\begin{array}{l}\text { Teneur en sable } \\
(\%)\end{array}$ & $\begin{array}{l}\text { Densité apparente } \\
\left(\mathrm{kg} \cdot \mathrm{dm}^{-3)}\right.\end{array}$ & $\begin{array}{l}\text { Matière organique } \\
(\%)\end{array}$ \\
\hline Leptosol lithique & & & & & \\
Effectif & 8 & 8 & 8 & 8 & 8 \\
Moyenne & 39 & 47 & 12 & 1,5 & 1,22 \\
Écart-type & 5,86 & 5,67 & 3,52 & 0,18 & 0,12 \\
\hline Régosol calcaire & & & & \\
Effectif & 5 & 5 & 5 & 5 & 5 \\
Moyenne & 28 & 40 & 3,05 & 1,43 & 0,08 \\
Écart-type & 3,75 & 3,56 & & & \\
\hline Cambisol & & & 3 & 3 & 3 \\
Effectif & 3 & 3 & 13 & 1,76 & 2,57 \\
Moyenne & 43 & 45 & 4,11 & 0,15 & 0,18 \\
Écart-type & 4,22 & 3,85 & & & \\
\hline
\end{tabular}

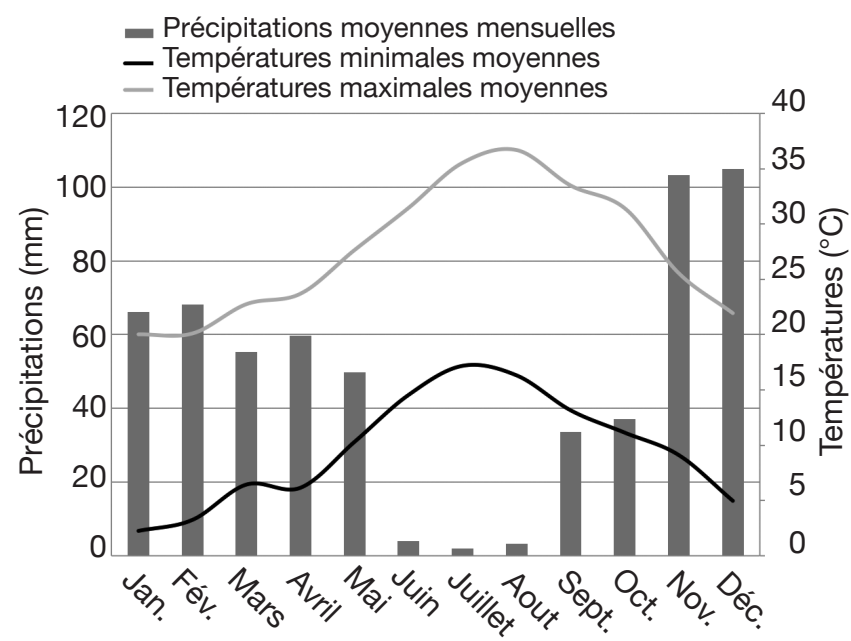

Figure 2. Précipitations et températures moyennes mensuelles à la station de Boukourdane - Average monthly precipitation and temperature at Boukourdane station (ANRH, 1988/1989 - 2012/2013).

allant de 1988 à 2012. La figure 3 illustre la variation interannuelle des précipitations et la moyenne annuelle.

Le barrage de Boukourdane. Le barrage de Boukourdane revêt un intérêt stratégique dans la politique algérienne d'autosuffisance alimentaire, tant en matière d'alimentation en eau potable des agglomérations situées aux alentours (Cherchell, Sidi Ghiles, Sidi Moussa, Sidi Amar, Nador et Tipasa), qu'en matière d'irrigation de la vallée de l'oued El Hachem et des régions de Hadjout et du Sahel.

Le barrage de Boukourdane a été construit en 1988 et mis sous eau en 1992. Sa capacité initiale était estimée à $93 \cdot 10^{6} \mathrm{~m}^{3}$ selon les plans originaux et a été

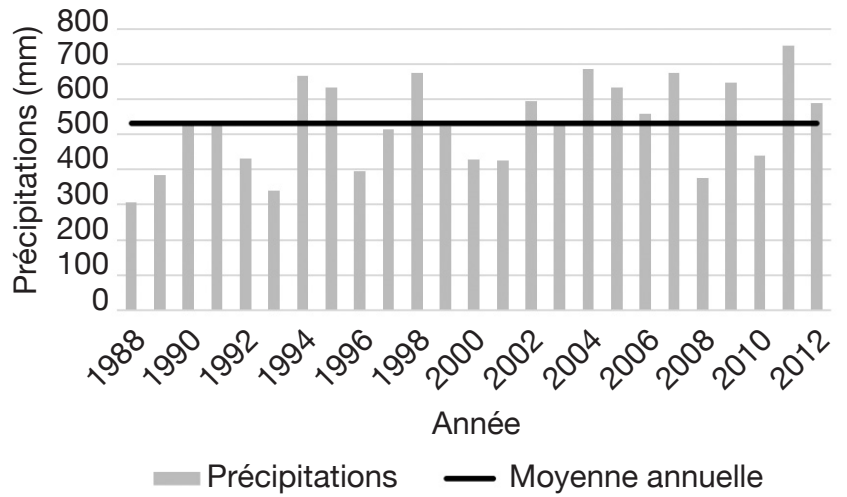

Figure 3. Variation interannuelle et moyenne annuelle des précipitations à la station de Boukourdane - Inter annual variation and monthly average of rainfall at Boukourdane station (1988/2013).

révisée en 2005, après réalisation de la bathymétrie à $108 \cdot 10^{6} \mathrm{~m}^{3}$. Sa construction a provoqué le rabattement de la nappe et même l'assèchement de certains puits situés à l'aval qui jusque-là bénéficiaient de la recharge naturelle de la nappe.

La protection de cet ouvrage contre un envasement rapide entrainant une diminution sensible de sa durée de vie et la gestion adéquate des lâchers de barrage sont des enjeux majeurs pour l'alimentation en eau potable de la région et l'irrigation. En effet, les lâchers du barrage permettent la réalimentation artificielle de la nappe phréatique qui alimente le champ captant.

Le champ captant. La plaine de l'oued El Hachem se situe au sud-est de la ville de Cherchell. Elle est limitée au Nord par la Mer Méditerranée (Figure 1). 
Tadrist N., Debauche O., Remini B. et al.

Elle était la principale ressource d'approvisionnement en eau potable de grandes villes telles que Tipaza et Cherchell, ainsi que l'irrigation de grandes parcelles agricoles.

L'aquifère de la nappe de l'oued El Hachem est d'âge Plio-Quaternaire. La nappe est tantôt libre, tantôt semi-captive, sous un recouvrement d'argile ou de marne d'épaisseur variable ne dépassant pas les $20 \mathrm{~m}$.

Toutes les études réalisées sur l'oued El Hachem montrent que la seule formation intéressante du point de vue hydrogéologique est représentée par le remplissage alluvionnaire du Quaternaire de la plaine. Ces dépôts du Quaternaire sont des galets, graviers, argiles sableuses, argiles rouges et limons de surface (Maamar, 2004).

Un réseau piézométrique de surveillance de la nappe alluviale de l'oued El Hachem (Figure 1) est constitué de 16 points de mesure qui se répartissent en 13 puits et 3 forages. La figure $\mathbf{4}$ montre la localisation de ces puits. La plupart de ces puits sont situés à proximité du lit de l'oued El Hachem à l'aval du barrage de

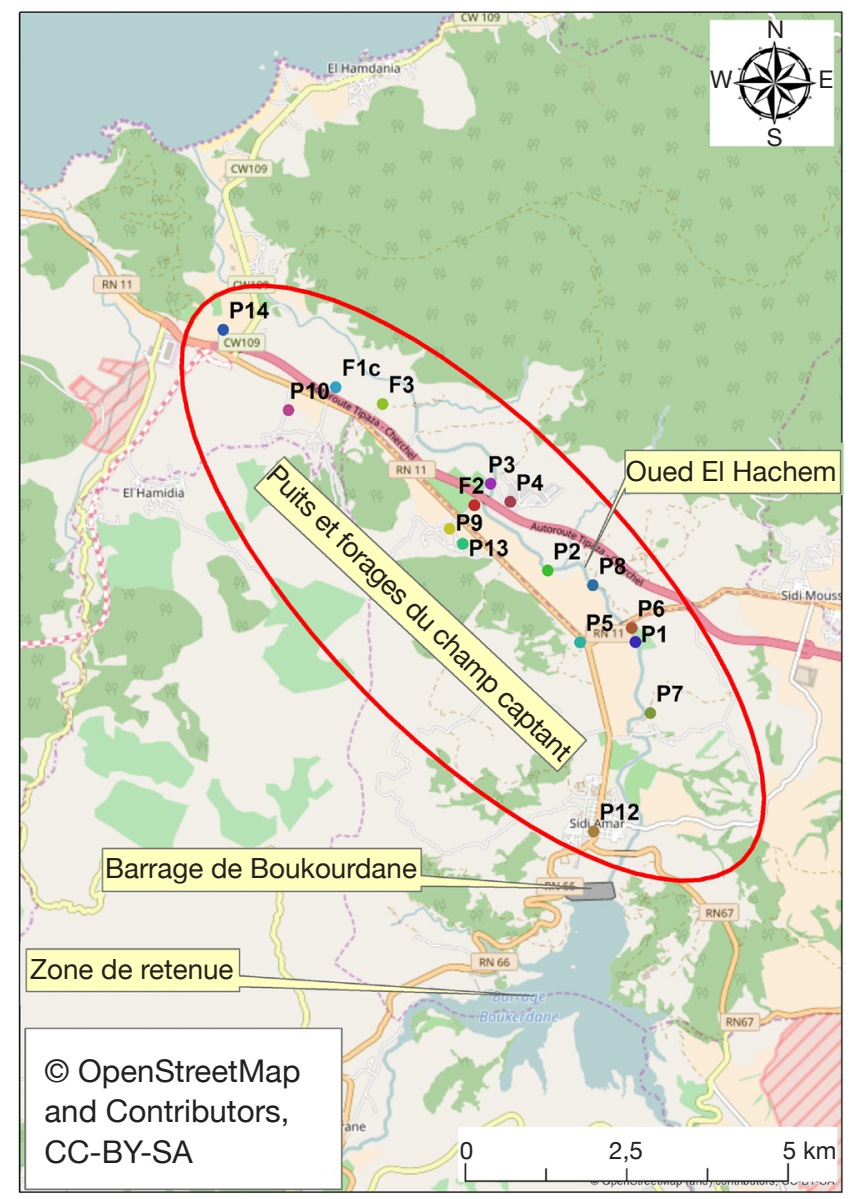

Figure 4. Localisation des puits $(\mathrm{P})$ et forages $(\mathrm{F})$ du champ captant par rapport au barrage de Bourkourdane - Location of wells $(P)$ and boreholes $(F)$ of the well field compared to the dam Bourkourdane.
Boukourdane. Des campagnes piézométriques ont été réalisées par l'Agence Nationale des Ressources Hydrauliques (ANRH) de 1998 à 2013.

\section{MÉTHODOLOGIE}

\section{1. Évaluation du transport des sédiments}

L'évaluation du transport des sédiments en suspension dans l'oued El Hachem a été effectuée au niveau de deux stations Bordj Ghobrini et Mesdour. La station de Bordj Ghobrini implantée sur l'oued El Hachem était en activité avant la construction du barrage de Boukourdane. Elle a été remplacée par la station de Mesdour après la construction du barrage. Cette station est située plus en amont sur l'oued Boukadir qui est le principal affluent de l'oued El Hachem.

L'échantillonnage de la concentration en matières solides en suspension, réalisé par les services de l'ANRH à la station de Mesdour et auparavant à la station de Bordj Ghobrini, a été effectué par prélèvement au bord de l'oued. Des mesures ponctuelles couvrent une période de 1993/1994 à 2012/2013.

La méthode consiste à effectuer des prélèvements d'échantillons d'eau chargée de sédiments en bordure pour différentes sections du cours d'eau. La concentration en sédiments exprimée en $\mathrm{g} \cdot \mathrm{l}^{-1}$ est obtenue au laboratoire après filtration à l'aide d'un filtre de marque FIORONI d'une porosité de 17 à $30 \mu \mathrm{m}$, séchage à $105^{\circ} \mathrm{C}$ durant $30 \mathrm{~min}$ et pesée des échantillons séchés.

L'apport solide en suspension As (g) est calculé par intégration du produit du débit solide en suspension $Q S$ $\left(\mathrm{g} \cdot \mathrm{s}^{-1}\right.$ ) dans le temps $t$ (s) (Yang et al., 2006 ; Moatar et al., 2007). Cependant, la mesure directe du débit solide n'est pas possible. En effet, le débit est évalué à partir de la courbe de tarage et de la lecture de la hauteur d'eau sur une échelle limnimétrique ; la concentration en matières en suspensions est déterminée a posteriori au laboratoire. L'apport solide se calcule en multipliant le débit liquide $Q_{L}\left(1 \cdot \mathrm{s}^{-1}\right)$ par la concentration $C\left(\mathrm{~g} \cdot \mathrm{l}^{-1}\right)$ :

$$
A s=\int_{0}^{t} C(t) \cdot Q(t) d t
$$

En considérant la variation linéaire du débit solide au pas de temps journalier, l'apport solide moyen pour la période entre les temps $t_{i}$ et $t_{i+1}$ est donné par la relation :

$$
A s_{i}=0,5 \cdot\left(Q_{i} C_{i}+Q_{i+1} C_{i+1}\right) \cdot\left(t_{i+1}-t_{i}\right)
$$

La somme arithmétique des apports solides élémentaires pendant une période donnée (mois, saison, année et crue) donnera l'apport solide de cette période. Le tonnage spécifique exprimé $t \cdot \mathrm{km}^{-2}$ est obtenu en 
divisant l'apport total par la surface du bassin versant $\left(81 \mathrm{~km}^{2}\right)$ drainée par la station de mesure.

Étant donné la quantité limitée de données disponibles actuellement, il ne nous est pas possible de recourir à la modélisation hydrologique à l'aide d'un modèle à base physique. En effet, les données météorologiques sont entachées de nombreuses erreurs, ne sont que rarement disponibles à l'échelle horaire et dans leur intégralité. La carte d'occupation du sol disponible est imprécise et comprend de nombreuses erreurs. Les données pédologiques précises ne sont disponibles que dans certaines zones. Généralement, c'est la carte au 1/500000 qui est utilisée. Dans la littérature, il existe plusieurs procédures de calcul de charge solide (Walling et al., 1981 ; Li et al., 2003 ; Clarke, 2009). Ces techniques font depuis toujours l'objet d'investigations en vue d'identifier la méthode qui évalue de la manière la plus fiable l'apport en suspension.

La technique la plus couramment utilisée est la régression. La qualité des relations obtenues est appréciée à l'aide de la valeur de $\mathrm{R}^{2}$.

Plusieurs modèles de régression sont utilisés pour le calcul du transport solide. Le modèle puissance de la forme $y=a x^{b}$ est souvent adopté pour la construction des courbes de production de sédiments où $y$ représente la variable dépendante $C$ (concentration) et $x$ représente la variable indépendante $Q_{L}$ (débit liquide). L'ajustement des paramètres du modèle puissance est obtenu par linéarisation en transformant $y$ en $\ln (y)$ et $x$ en $\ln (x)$. Ce modèle est appliqué pour la première fois par Kennedy en 1895 pour le couple (débit liquide-concentration) et ensuite Mimikou (1982), Horowitz (2002) et Ghenim (2008) l'ont appliqué pour le couple (débit liquidedébit solide).

Le modèle de régression de type puissance est justifié par plusieurs chercheurs tels que Bourouba (1997), Megnounif et al. (2003), Achite et al. (2007), Khanchoul et al. (2012) et Megnounif et al. (2013). Ils constatent que ce modèle offre un bon ajustement et permet d'évaluer les valeurs journalières du débit solide $Q_{S}$ à partir des débits liquides $Q_{L}$ observés, d'étudier les relations débit liquide-débit solide et l'influence des débits liquides sur le transport solide à différentes échelles temporelles : annuelle, mensuelle et saisonnière (Achite et al., 2004).

\section{2. Évaluation de la recharge du champ captant}

L'évaluation de la recharge du champ captant est effectuée sur base de relevés piézométriques réalisés par l'ANRH la semaine qui précède et celle qui suit chaque campagne de recharge de la nappe phréatique par lâchers du barrage de Boukourdane, qui ont lieu en moyenne deux fois par an, généralement en mai et octobre. L'objectif de l'étude est de mettre en évidence l'impact des particules fines accumulées lors des lâchers sur la recharge de la nappe phréatique. Les puits et forages ont été rassemblés en groupes par la méthode des k-moyennes à l'aide du logiciel libre Weka. La méthode des k-moyennes (k-means) est une méthode de classification supervisée permettant de partitionner des données. Un ensemble de puits et forages sont séparés en k groupes (clusters) sur base d'un ensemble de caractéristiques (variation piézométrique avant et après lâcher de barrage, distance par rapport au barrage, distance par rapport à l'oued) (Bouckaert, 2010). Cet algorithme consiste à minimiser la distance moyenne quadratique à partir de chaque point de données par rapport au centre du groupe le plus proche. L'algorithme s'exprime mathématiquement comme suit :

$$
{ }^{\arg } S^{\min } \sum_{i=1}^{k} \sum_{x_{j} \in S_{i}}\left\|x_{j}-\mu_{i}\right\|^{2}
$$

où $\left(\mathrm{x}_{1}, \mathrm{x}_{2}, \ldots, \mathrm{x}_{\mathrm{n}}\right)$ est un ensemble de $n$ points, $k$ est le nombre de partitionnements, $S$ est un ensemble de $k$ partitionnements $\left(\mathrm{S}_{1}, \mathrm{~S}_{2}, \ldots \mathrm{S}_{\mathrm{k}}\right)$ des $n$ points avec $k \leq n$, $\mu_{\mathrm{i}}$ est la moyenne des points dans la partition $\mathrm{S}_{\mathrm{i}}$.

L'heuristique k-moyennes a été utilisé afin de regrouper les puits et les forages en fonction de la distance par rapport au barrage et de la distance par rapport au lit de l'oued. L'efficacité de cet algorithme a par ailleurs été démontrée par Abedini et al. (2008) lors de la comparaison de k-moyenne avec le krigeage ordinaire.

Sur base du regroupement des puits et forages, des courbes d'ajustement linéaire pour les parties amont et aval et d'ajustement de puissance pour la partie centrale du champ captant ont été tracées à partir d'une série chronologique de variations piézométriques moyennes au sein de chaque groupe. Ces courbes d'ajustement permettent d'étudier l'évolution de la perméabilité du champ captant en fonction du temps. Les niveaux piézométriques en fonction du temps sont en relation avec le champ de perméabilité (Degallier, 1969; Ungemach, 1969).

\section{RÉSULTATS}

Comme le montre le tableau 3, l'analyse statistique réalisée à différentes échelles temporelles a permis de montrer que la relation puissance était quasi systématiquement le meilleur modèle prédictif de la relation débit solide $\left(Q_{S}\right)$ - débit liquide $\left(Q_{L}\right)$. Le tableau 3 présente les relations obtenues pour différentes échelles temporelles (série complète, automne, hiver, printemps, été, période sèche, période humide, période de crues) et les coefficients de corrélation associés. 
Tableau 3. Relations et coefficients de corrélation des débits solides $\left(Q_{S}\right)$ et débits liquides $\left(Q_{L}\right)$ à la station de Mesdour - Relationships and correlation coefficients of solid $\left(\mathrm{Q}_{\mathrm{S}}\right)$ and liquid $\left(\mathrm{Q}_{\mathrm{L}}\right)$ discharges at Mesdour station.

\begin{tabular}{|c|c|c|c|c|c|}
\hline Période & Puissance & Exponentielle & Linéaire & Logarithmique & Polynomiale \\
\hline \multicolumn{6}{|c|}{ Série complète } \\
\hline $\begin{array}{l}Q_{S} \\
\mathrm{R}^{2} \\
\end{array}$ & $\begin{array}{l}2,5182 Q_{L}^{0,8193} \\
0,77\end{array}$ & $\begin{array}{l}3,263 \mathrm{e}^{0,0182} Q_{L} \\
0,13\end{array}$ & $\begin{array}{l}0,4204 Q_{L}+18,102 \\
0,25\end{array}$ & $\begin{array}{l}8,4987 \ln \left(Q_{L}\right)+19,114 \\
0,29\end{array}$ & $\begin{array}{l}-0,0012 Q_{L}^{2}+0,7701 Q_{L}+15,53 \\
0,26\end{array}$ \\
\hline \multicolumn{6}{|c|}{ Automne } \\
\hline $\begin{array}{l}Q_{S} \\
\mathrm{R}^{2}\end{array}$ & $\begin{array}{l}1,1663 Q_{L}^{2,0026} \\
0,76\end{array}$ & $\begin{array}{l}2,6657 \mathrm{e}^{0,306} Q_{L} \\
0,33\end{array}$ & $\begin{array}{l}10,702 Q_{L}+65,302 \\
0,15\end{array}$ & $\begin{array}{l}47,895 \ln \left(Q_{L}\right)+72,511 \\
0,16\end{array}$ & $\begin{array}{l}-0,5712 Q_{L}^{2}+24,367 Q_{L}+11,253 \\
0,17\end{array}$ \\
\hline \multicolumn{6}{|l|}{ Hiver } \\
\hline $\begin{array}{l}Q_{S} \\
\mathrm{R}^{2}\end{array}$ & $\begin{array}{l}1,3383 Q_{L}^{1,1962} \\
0,80\end{array}$ & $\begin{array}{l}1,0897 \mathrm{e}^{0,2373} Q_{L} \\
0,54\end{array}$ & $\begin{array}{l}2,0275 Q_{L}+5,8603 \\
0,30\end{array}$ & $\begin{array}{l}9,716 \ln \left(Q_{L}\right)+8,2496 \\
0,40\end{array}$ & $\begin{array}{l}-0,2205 Q_{L}^{2}+6,2611 Q_{L}-4,7758 \\
0,54\end{array}$ \\
\hline \multicolumn{6}{|c|}{ Printemps } \\
\hline $\begin{array}{l}Q_{S} \\
\mathrm{R}^{2}\end{array}$ & $\begin{array}{l}0,4105 Q_{L}^{1,0643} \\
0,94\end{array}$ & $\begin{array}{l}3,3571 \mathrm{e}^{0,0288} Q_{L} \\
0,70\end{array}$ & $\begin{array}{l}0,5651 Q_{L}+0,367 \\
0,94\end{array}$ & $\begin{array}{l}15,42 \ln \left(Q_{L}\right)-21,283 \\
0,69\end{array}$ & $\begin{array}{l}-0,0008 Q_{L}^{2}+0,6694 Q_{L}-1,8251 \\
0,95\end{array}$ \\
\hline \multicolumn{6}{|l|}{ Été } \\
\hline $\begin{array}{l}Q_{S} \\
\mathrm{R}^{2}\end{array}$ & $\begin{array}{l}0,1576 Q_{L}^{0,3094} \\
0,53\end{array}$ & $\begin{array}{l}0,0163 \mathrm{e}^{121,09} Q_{L} \\
0,72\end{array}$ & $\begin{array}{l}3,3861 Q_{L}+0,0141 \\
0,76\end{array}$ & $\begin{array}{l}0,0087 \ln \left(Q_{L}\right)+0,0776 \\
0,56\end{array}$ & $\begin{array}{l}515,54 Q_{L}^{2}-0,7102 Q_{L}+0,0199 \\
0,83\end{array}$ \\
\hline \multicolumn{6}{|c|}{ Période humide } \\
\hline $\begin{array}{l}Q_{S} \\
\mathrm{R}^{2}\end{array}$ & $\begin{array}{l}1,3634 Q_{L}^{1,4292} \\
0,72\end{array}$ & $\begin{array}{l}6,5786 \mathrm{e}^{0,0293} Q_{L} \\
0,12\end{array}$ & $\begin{array}{l}0,1825 Q_{L}+50,155 \\
0,003\end{array}$ & $\begin{array}{l}13,302 \ln \left(Q_{L}\right)+29,616 \\
0,05\end{array}$ & $\begin{array}{l}-0,0065 Q_{L}^{2}+0,919 Q_{L}+44,472 \\
0,008\end{array}$ \\
\hline \multicolumn{6}{|c|}{ Période sèche } \\
\hline $\begin{array}{l}Q_{S} \\
\mathrm{R}^{2} \\
\end{array}$ & $\begin{array}{l}1,8969 Q_{L}^{0,6838} \\
0,55\end{array}$ & $\begin{array}{l}0,1446 \mathrm{e}^{0.0641} Q_{L} \\
0,98\end{array}$ & $\begin{array}{l}0,5677 Q_{L}+0,1439 \\
0,97\end{array}$ & $\begin{array}{l}4,2457 \ln \left(Q_{L}\right)+20,833 \\
0,60\end{array}$ & $\begin{array}{l}-0,0005 Q_{L}^{2}+0,6287 Q_{L}-0,3806 \\
0,97\end{array}$ \\
\hline \multicolumn{6}{|l|}{ Crues } \\
\hline $\begin{array}{l}Q_{S} \\
\mathrm{R}^{2}\end{array}$ & $\begin{array}{l}2,2746 Q_{L}^{0,7839} \\
0,78\end{array}$ & $\begin{array}{l}2,5984 \mathrm{e}^{0,0186} Q_{L} \\
0,16\end{array}$ & $\begin{array}{l}0,431 Q_{L}+10,645 \\
0,61\end{array}$ & $\begin{array}{l}6,6643 \ln \left(Q_{L}\right)+13,943 \\
0,41\end{array}$ & $\begin{array}{l}-0,0007 Q_{L}^{2}+0,6337 Q_{L}+9,2131 \\
0,63\end{array}$ \\
\hline
\end{tabular}

Le tableau 4 montre les quantités d'érosion mensuelles calculées à partir du meilleur modèle (puissance établi sur la série complète) et des débits liquides mesurés.

La figure 5 illustre les relations obtenues par le meilleur modèle (puissance) pour les différentes échelles temporelles.

La figure 6 présente l'évolution mensuelle et interannuelle des apports solides.

Les données expérimentales montrent que de forts débits solides allant jusqu'à $179,64 \mathrm{~kg} \cdot \mathrm{s}^{-1}$ peuvent être associés avec de faibles débits liquides (inférieurs à $\left.16 \mathrm{~m}^{3} \cdot \mathrm{s}^{-1}\right)$ déclenchés probablement à la suite d'orages violents et de courte durée. On remarque un flux important de matières solides en suspension en dehors des mois d'été. Le débit solide maximum a été atteint en novembre 1996 avec $134 \mathrm{~kg} \cdot \mathrm{s}^{-1}$ et un débit liquide de $4,6 \mathrm{~m}^{3} \cdot \mathrm{s}^{-1}$. En revanche, on constate une diminution nette durant la saison estivale des débits liquides qui ne dépassent pas les $7 \mathrm{~m}^{3} \cdot \mathrm{s}^{-1}$ à l'étiage.

Au début de l'automne, les premières précipitations tombent sur un sol sec et dur. Elles contribuent à la rupture des liaisons entre les particules du sol, mais sans produire une érosion importante. Le transport solide en suspension dans le bassin versant est donc faible.

Au milieu et à la fin de l'automne, le régime pluviométrique change. Des pluies torrentielles font leur apparition et arrachent de grandes quantités de matières solides qui seront par la suite transportées en suspension par le cours d'eau. Des ruissellements importants ainsi que des écoulements hypodermiques font leur apparition durant cette période sur l'entièreté du bassin versant.

\subsection{Bilan des apports solides}

Pour estimer les apports solides en suspension, nous avons établi la relation suivante sur base de la série de données complète :

$$
Q_{S}=2,6053 \cdot Q_{L}^{0,8216}
$$

où $Q_{S}$ est le débit solide $\left(\mathrm{kg} \cdot \mathrm{s}^{-1}\right)$ et $Q_{L}$ est le débit liquide $\left(\mathrm{m}^{3} \cdot \mathrm{s}^{-1}\right)$. 


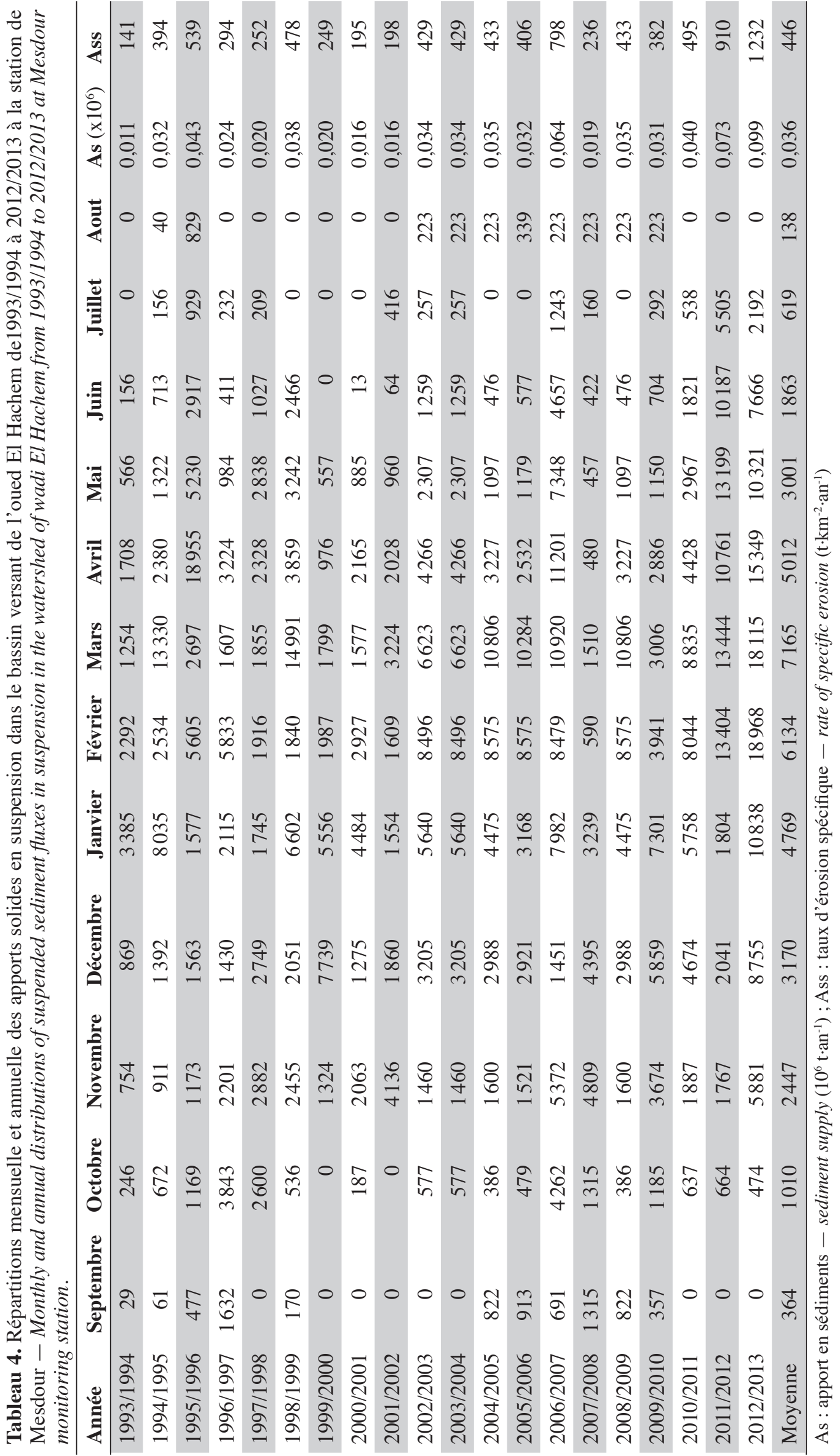


Série complète

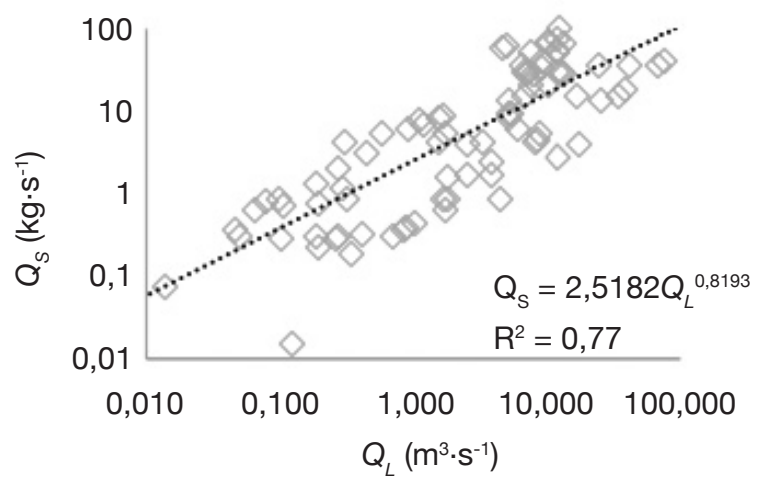

Période humide

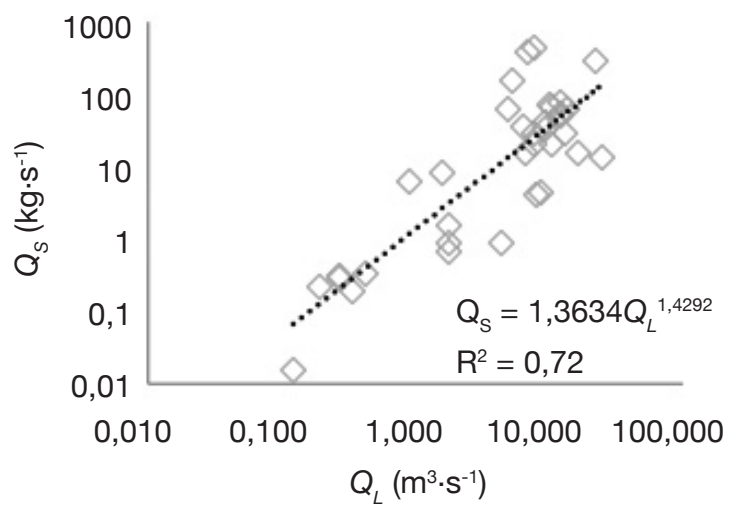

Automne

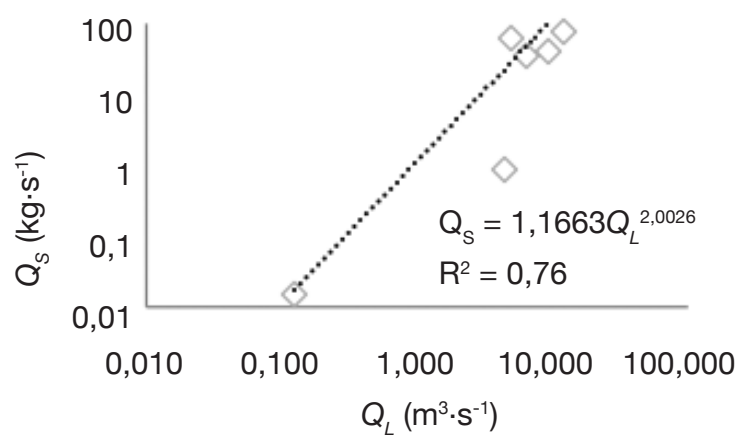

Printemps

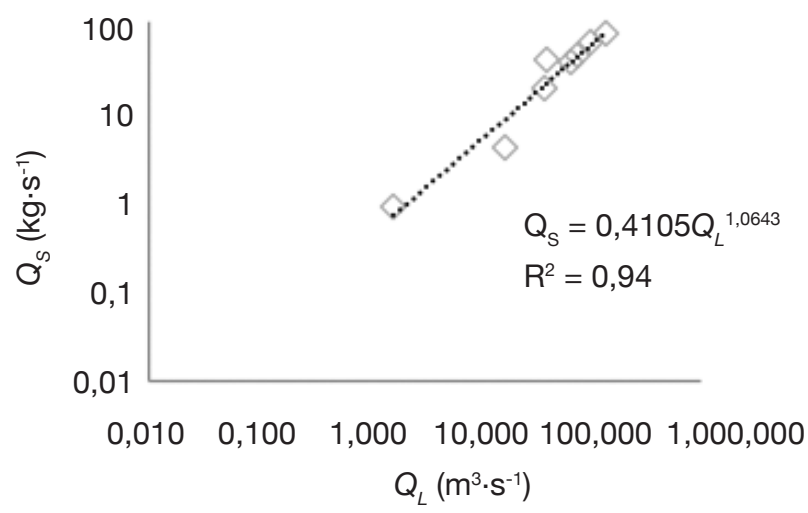

Crues

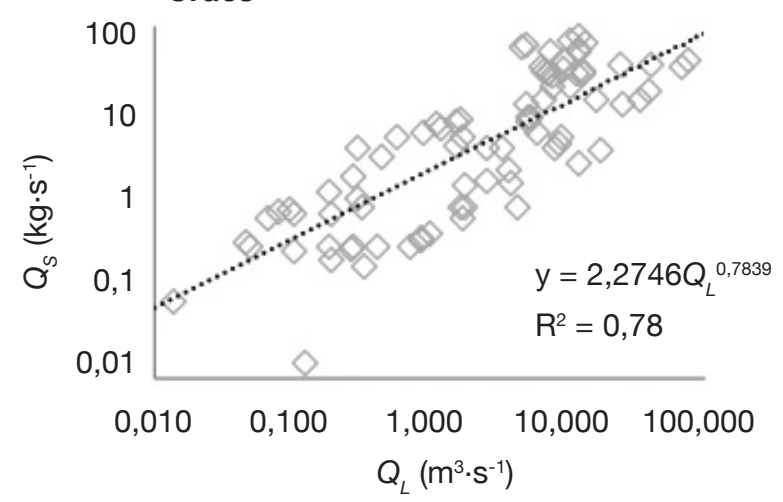

Période sèche

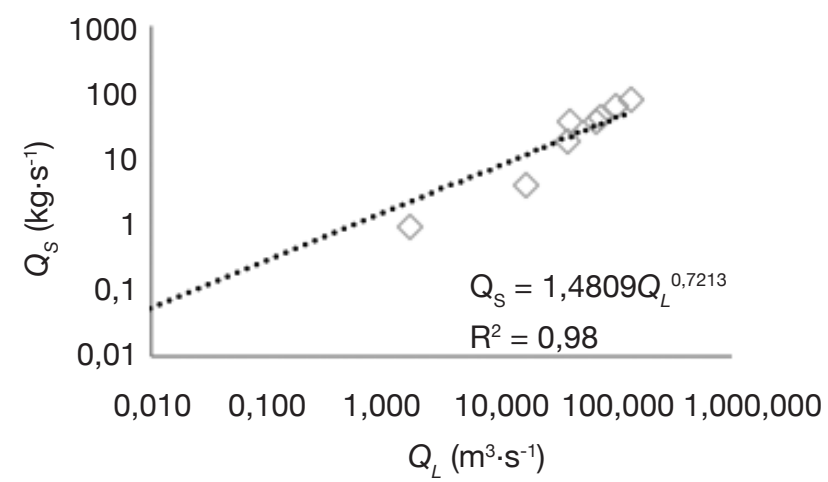

Hiver

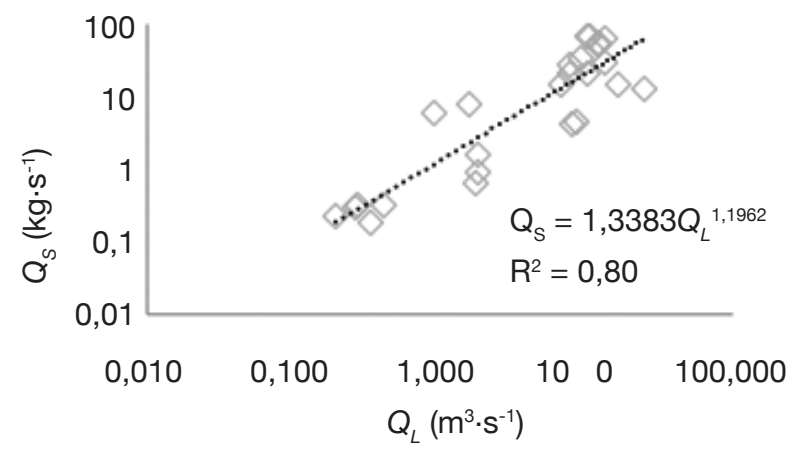

Été

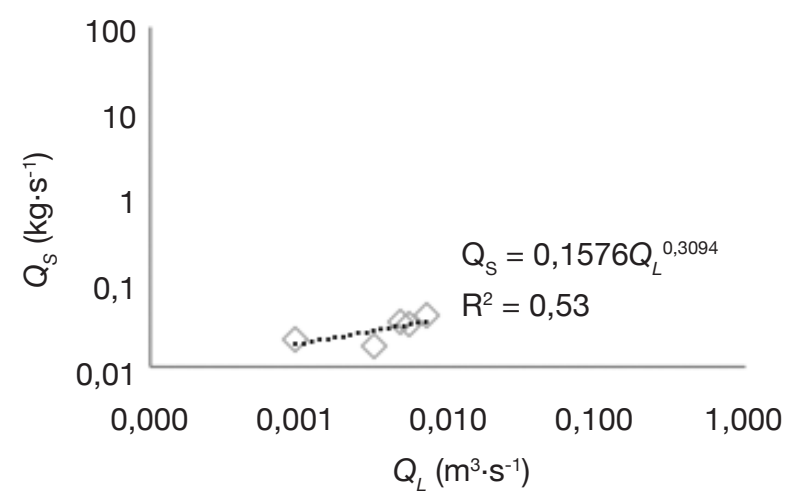

Figure 5. Relations entre débits solides $\left(Q_{S}\right)$ et débits liquides $\left(Q_{L}\right)$ instantanés mesurés entre 1993 et 2013 sur l'oued El Hachem - Relationships between instantaneous sediment and river water discharge $\left(\mathrm{Q}_{\mathrm{S}}\right)$ and liquid flow $\left(\mathrm{Q}_{\mathrm{L}}\right)$ measured between 1993 and 2013 on wadi El Hachem. 


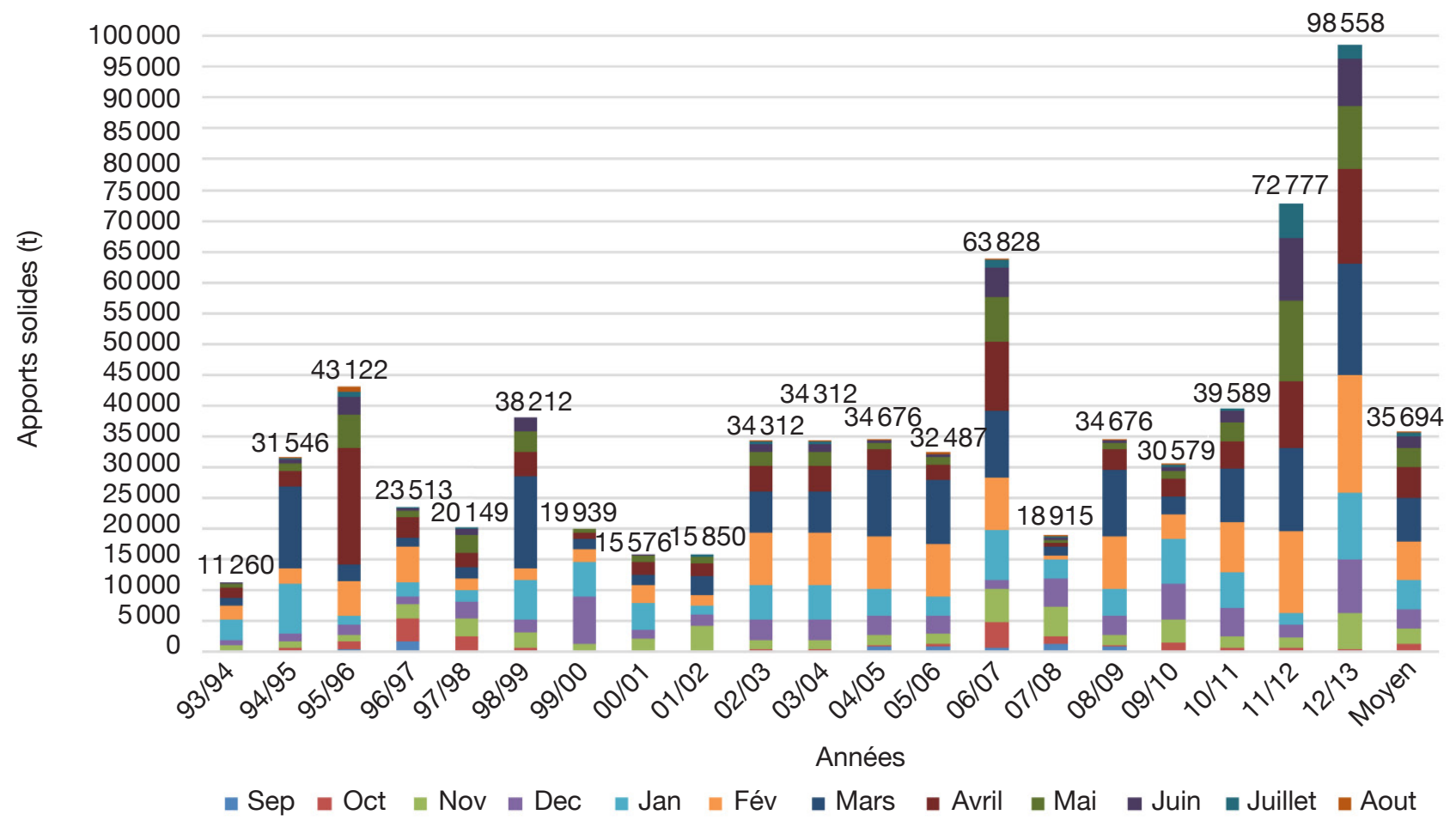

Figure 6. Répartitions mensuelle et interannuelle des apports solides en suspension dans le bassin versant de l'oued El Hachem de 1993/1994 à 2012/2013 à la station de Mesdour - Monthly and inter annual distributions of suspended sediment fluxes in the watershed of wadi El Hachem from 1993/1994 to 2012/2013 at Mesdour monitoring station.

Le calcul a été également réalisé à l'échelle annuelle, interannuelle, saisonnière sur une période de 20 ans allant de 1993/1994 à 2012/2013. Les résultats obtenus sont repris dans le tableau 4 et illustrés à la figure 6.

Le tableau 4 montre que les années 1995/1996 et 1998/1999 se caractérisent par un apport en sédiments plus élevé, comparées aux autres années. Ces concentrations élevées s'expliquent par une succession de crues exceptionnelles durant cette période. On notera plus particulièrement les crues d'avril 1995, mars 1998, mars 2004, avril 2006 et mars 2009, de février à mai 2012, et de janvier à mai 2013 : des apports solides respectivement de $18955 \mathrm{t}$, $14991 \mathrm{t}, 10806 \mathrm{t}, 11202 \mathrm{t}, 10807 \mathrm{t}$, compris entre 10187 et $13444 \mathrm{t}$ et compris entre 10321 et $18968 \mathrm{t}$ ont été calculés correspondant à des apports liquides respectifs de $8,77 \cdot 10^{6} \mathrm{~m}^{3} ; 6,81 \cdot 10^{6} \mathrm{~m}^{3} ; 4,53 \cdot 10^{6} \mathrm{~m}^{3}$; $4,77 \cdot 10^{6} \mathrm{~m}^{3}$ et $3,57 \cdot 10^{6} \mathrm{~m}^{3}$.

On constate, par ailleurs, que les quantités importantes de sédiments transportés en suspension par l'oued El Hachem sont principalement observées entre les mois de décembre à avril. Cette analyse a été confirmée par d'autres auteurs sur des oueds voisins (Achite et al., 2005 ; Elahcène et al., 2013 ; Megnounif et al., 2013).

Les dernières années 2011-2012 et 2012-2013 ont été marquées par des crues exceptionnelles durant les mois de février à mai qui se sont traduites par une érosion particulièrement importante (Tableau 4). Le flux moyen annuel des sédiments calculé au droit de la station de Mesdour est de 35693 t.an ${ }^{-1}$. L'apport en sédiments au niveau du barrage de Boukourdane est de 69602 t. Cet apport s'obtient par extrapolation du taux d'érosion spécifique des sols de $446 \mathrm{t} \cdot \mathrm{km}^{-2} \cdot \mathrm{an}^{-1}$.

La moyenne d'érosion spécifique calculée pour la station hydrométrique Bordj Ghobrini était de $340 \mathrm{t}$. $\mathrm{km}^{-2} \cdot \mathrm{an}^{-1}$ (Tadrist, 2004). Cette valeur est très proche de la valeur moyenne à la station de Mesdour de $366 \mathrm{t}$. $\mathrm{km}^{-2} \cdot \mathrm{an}^{-1}$ calculée avec les données de 1993 à 2005. On peut donc faire l'hypothèse que les mesures effectuées à la station de Mesdour sont représentatives pour le bassin versant anciennement jaugé par la station de Bordj Ghobrini.

Comme le montre le tableau 1, le taux d'érosion spécifique des sols trouvé pour l'oued Boukadir est proche de celui trouvé par d'autres auteurs pour des bassins versants de caractéristiques similaires. Comme le montre le tableau 5, le taux d'envasement du barrage de Boukourdane mesuré par la bathymétrie de 2005 de l'Agence Nationale des Barrages et Transferts (ANBT) est similaire à celui que nous avons calculé. En effet, le taux de dégradation spécifique (Ass) à Mesdour est de $366 \mathrm{t} \cdot \mathrm{km}^{-2} \cdot \mathrm{an}^{-1}$ en moyenne pour la période de 1993 à 2005 et de $446 \mathrm{t} \cdot \mathrm{km}^{-2} \cdot \mathrm{an}^{-1}(+22 \%)$ en moyenne pour une période de 1993 à 2013. En 
Tableau 5. Taux d'envasement évalués par l'ANBT en 2005 pour les barrages de la région du centre de l'Algérie - Siltation rate assessed by ANBT in 2005 for dams in the region of central Algeria.

\begin{tabular}{lccccc}
\hline Barrages & $\begin{array}{l}\text { Capacité initiale } \\
\left(10^{6} \mathrm{~m}^{3}\right)\end{array}$ & $\begin{array}{l}\text { Capacité dernier } \\
\text { levé }\left(10^{6} \mathrm{~m}^{3}\right)\end{array}$ & $\begin{array}{l}\text { Envasement moyen } \\
\text { annuel }\left(10^{6} \mathrm{~m}^{3}\right)\end{array}$ & $\begin{array}{l}\text { Envasement dernier } \\
\text { levé }\left(10^{6} \mathrm{~m}^{3}\right)\end{array}$ & $\begin{array}{l}\text { Taux d'envase- } \\
\text { ment }(\%)\end{array}$ \\
\hline Bouroumi & 188,00 & 181,86 & 0,31 & 6,14 & 3,27 \\
Meurad & 0,90 & 0,20 & 0,00 & 0,70 & 77,78 \\
Ladrat & 10,00 & 8,47 & 0,10 & 1,53 & 15,30 \\
Boukourdane & $\mathbf{1 0 8 , 0 0}$ & $\mathbf{1 0 7 , 0 0}$ & $\mathbf{0 , 0 8}$ & $\mathbf{1 , 0 0}$ & $\mathbf{1 , 0 3}$ \\
Keddara & 145,60 & 142,39 & 0,17 & 3,21 & 2,20 \\
Beni-Amrane & 16,00 & 11,85 & 0,24 & 4,15 & 25,94 \\
Hamiz & 21,00 & 15,53 & 0,08 & 5,47 & 26,05 \\
Lekhal & 30,00 & 27,16 & 0,14 & 2,84 & 9,47 \\
Tilesdit & 167,00 & 164,55 & 2,45 & 2,45 & 1,47 \\
\hline
\end{tabular}

considérant une masse volumique des sédiments de $1,6 \mathrm{~kg} \cdot \mathrm{dm}^{-3}$ et une superficie de bassin versant de $156 \mathrm{~km}^{2}$, nous obtenons des volumes de sédiments produits annuellement respectivement de $8,38 \cdot 10^{4} \mathrm{~m}^{3}$ pour la période 1993 à 2005 et de $11,1 \cdot 10^{4} \mathrm{~m}^{3}$ pour la période 1993 à 2013. Cette augmentation du volume d'envasement est vraisemblablement due à la série de pluies exceptionnelles qu'a connues l'Algérie ces dernières années (Touaibia, 2010). En effet, aucune évolution de l'occupation du sol ou des pratiques culturales n'a été enregistrée durant cette période dans le bassin versant.

\subsection{Bilan de la recharge du champ captant}

Avant la mise en eau du barrage en 1992, la nappe alluviale en aval du barrage Boukourdane a toujours bénéficié d'une recharge naturelle par le biais des eaux de ruissellement issues des précipitations et des apports de l'oued El Hachem.

Cependant, la baisse sensible de la pluviométrie ces deux dernières décennies, associée au remplissage du barrage, ont contribué à l'augmentation des volumes soutirés de la nappe en entrainant une baisse des niveaux piézométriques. Des mesures de niveaux piézométriques au droit des puits et forages situés à l'aval du barrage ont fait l'objet d'un suivi depuis les premiers lâchers, le 1 avril 1998 jusqu'à ce jour. La figure 4 montre la répartition des puits et forages sur le champ captant. L'algorithme k-moyennes a été utilisé pour effectuer un regroupement sur base de la distance entre le puits ou le forage et le barrage, d'une part et la variation piézométrique moyenne, d'autre part. Le regroupement optimum consiste à regrouper les puits et forages en trois groupes distincts. Ce nombre de groupes correspond au point d'inflexion de la courbe d'erreur en fonction du nombre de groupes. Le groupe 1 contient les puits et forage P7, P1, P6, P5 et $\mathrm{P} 8$. Le groupe 2 rassemble les puits $\mathrm{P} 2, \mathrm{P} 4, \mathrm{P} 13$, $\mathrm{F} 2$ et $\mathrm{P} 9$. Le groupe 3 associe le puits et les forages : F3, F1 et P10. Sur base de ces groupes, les courbes d'ajustement illustrées à la figure 7 ont été calculées à partir des variations de hauteurs piézométriques moyennes après lâchers.

Comme le montre la figure 7, le champ captant se colmate de manière continue et progressive de l'amont vers l'aval et tend à devenir complètement imperméable (variation piézométrique nulle ou négative traduisant un déficit). L'imperméabilisation continue est vraisemblablement due à la formation d'une croute épaisse de particules fines provenant du barrage et remises en suspension lors des lâchers.

L'ensemble du champ captant est touché à des degrés divers par le dépôt de matières en suspension. La partie amont représentée par le groupe 1 est principalement le siège de dépôt des particules les plus grossières et est sujette à une diminution de la recharge de la nappe phréatique plus rapide que le reste du champ captant. Les particules les plus fines se déposent préférentiellement dans les parties centrale et aval du champ captant. La diminution de la recharge y est moins rapide. À partir de 2010, la gestion en matière de lâchers a été modifiée pour passer d'un seul lâcher à plusieurs lâchers et en 2012, un lâcher important de $10,2 \cdot 10^{6} \mathrm{~m}^{3}$ a été réalisé du 01/03/2012 au 30/04/2012, ce qui correspond à un débit moyen de $1,96 \mathrm{~m}^{3} \cdot \mathrm{s}^{-1}$. Cette modification de la politique de lâchers a eu pour conséquence une restauration partielle de la perméabilité et une amélioration de la recharge au niveau de la partie centrale du champ captant (groupe 2). Aucun impact marqué n'a été observé dans la partie amont (groupe 1) et aval (groupe 3). 
Groupe 1 (amont)

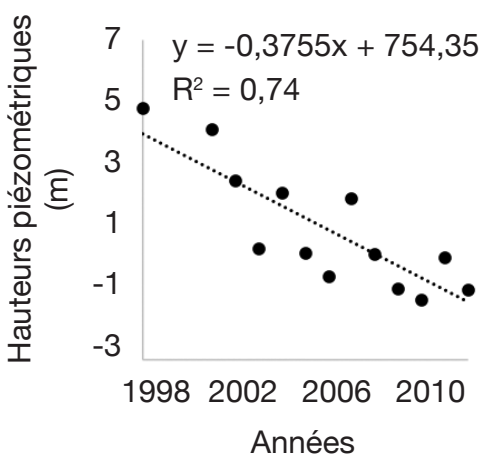

Groupe 2 (centre)

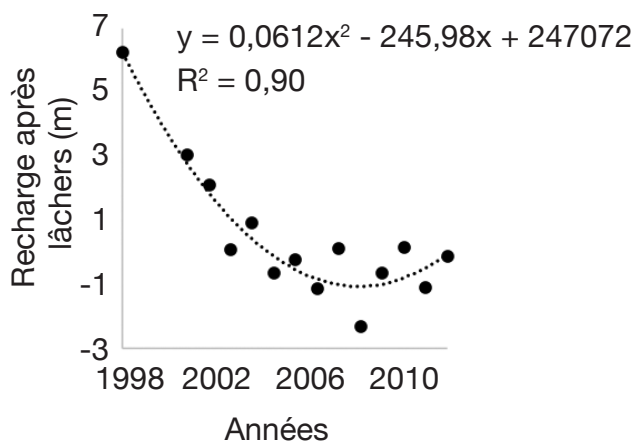

Groupe 3 (aval)

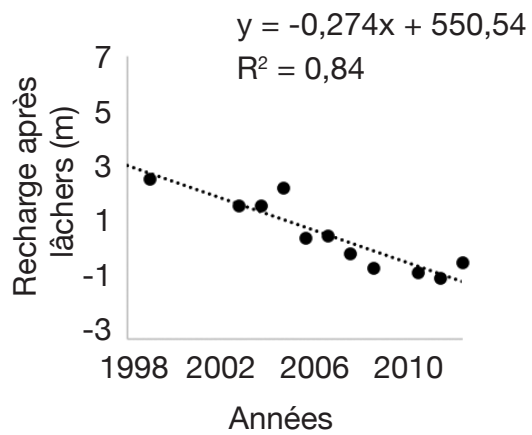

Figure 7. Fluctuation des hauteurs piézométriques après lâchers de barrage et localisation des puits et forages — Fluctuation of the water table height after dam releases and location of wells and boreholes.

\section{DISCUSSION}

L'implantation du barrage de Boukourdane en 1988 a modifié considérablement l'hydrologie de la zone d'étude. La recharge qui s'effectuait naturellement par les apports de l'oued El Hachem n'existe plus. Les conséquences ont été un abattement important de la nappe et une pénurie d'eau au niveau du champ captant situé en aval du barrage. Des lâchers de barrages ont ensuite été effectués pour tenter de compenser la recharge naturelle qui existait avant l'implantation du barrage. Cependant, ces lâchers ont une incidence négative sur la conductivité hydraulique du champ captant. L'accumulation successive des particules fines remises en suspension lors des lâchers a provoqué au fil du temps un colmatage du champ captant. En effet, lors de la percolation, les particules les plus fines sont entrainées et provoquent le colmatage de la macroporosité du sol.

Au travers de cette étude, nous avons quantifié les apports en matière en suspension arrivant au niveau du barrage et montré la diminution progressive de la recharge de la nappe lors des lâchers.

Le transport solide en suspension du bassin versant de l'oued El Hachem est particulièrement actif durant les périodes de crues. Les crues ont lieu principalement en hiver et dans une moindre mesure en automne et au printemps.

Les débits solides en suspension sont étroitement liés aux débits liquides. L'étude des relations empiriques à différentes échelles temporelles a confirmé que le modèle d'ajustement puissance était le meilleur.

Sur base de la relation $n^{\circ} 3$ ( $§ 4.1$.), nous avons calculé le débit solide en suspension journalier pour une période de référence de 20 ans allant de 1993/1994 à 2012/2013. Les résultats obtenus ont permis de calculer le tonnage annuel des sédiments transportés en suspension par l'oued El Hachem. L'apport moyen annuel en sédiments enregistré au niveau de la station de Mesdour est de 35693 t qui correspond à un taux d'érosion spécifique de $446 \mathrm{t} \cdot \mathrm{km}^{-2} \cdot \mathrm{an}^{-1}$. Cette valeur est concordante avec les valeurs trouvées notamment par Achite et al. (2007), Khanchoul et al. (2007), Khanchoul et al. (2012), Elahcene et al. (2013) et Megnounif et al. (2013) pour des bassins versants de même taille au Maghreb.

Les campagnes de suivi piézométrique ont montré une diminution substantielle de la recharge de la nappe phréatique au cours de ces dernières années. Cette diminution est vraisemblablement due à l'accumulation de particules fines qui sont transportées vers le champ captant lors des lâchers du barrage de Boukourdane. Il est donc important de restaurer la conductivité hydraulique du sol au plus vite afin de limiter les effets de rabattement de la nappe liés à la surexploitation du champ captant et éviter que l'une des dernières nappes phréatiques non encore contaminée par des intrusions marines ne le soit dans un proche avenir. Par ailleurs, Abdalla et al. (2013) ont montré que la recharge artificielle par lâchers de barrage en milieu aride était un moyen de limiter les intrusions marines. Des constatations similaires ont été effectuées par Prathapar et al. (2014). Ces auteurs ont mis en évidence que l'accumulation de sédiments réduisait le taux de recharge et les volumes de recharges. Ils ont également montré que l'évacuation régulière des sédiments permettait de restaurer la conductivité hydraulique et d'accélérer la recharge de la nappe. L'évacuation des sédiments devra être complétée par ratissage de la couche de sédiments accumulés et l'enlèvement de la couche superficielle du sol (Prathapar et al., 2014).

\section{CONCLUSIONS}

Ce travail a permis d'estimer la production de sédiments dans le bassin versant alimentant le barrage de Boukourdane à $11 \cdot 10^{4} \mathrm{~m}^{3} \cdot \mathrm{an}^{-1}$. Il a également 
montré que l'accumulation des sédiments au niveau du champ captant a provoqué une diminution continue de la transmissivité. La restauration de la transmissivité du champ captant ne pourra être réalisée qu'en changeant drastiquement la manière dont sont conduits actuellement les lâchers de barrage.

Un décapage du champ captant sera sans doute nécessaire pour restaurer complètement sa transmissivité. En effet, les particules fines ont, durant la percolation, colmaté la macroporosité du sol, provoquant ainsi son imperméabilisation progressive. Il est évident que des lâchers important ne permettront pas l'évacuation complète des particules fines.

On est également en droit de s'interroger sur le fait qu'aucun débit écologique minimal n'est lâché en continu durant l'année. Ne serait-ce pas une manière différente de faire de la recharge de nappe en continu tout en restaurant l'écosystème aquatique présent avant la construction du barrage de Boukourdane ?

Une modélisation hydrologique à l'aide d'un modèle à base physique permettrait de déterminer le débit écologique soutenable à assurer, tout en conservant un volume de stockage permanent. Toutefois, des données régulières et de qualité des précipitations, températures, vitesses du vent, radiation solaire ne sont généralement pas toutes disponibles à une échelle plus fine que l'échelle journalière. Quant aux mesures de débit liquide et de concentration en sédiments, elles ne sont pour l'heure mesurées que ponctuellement. Les données cartographiques précises en matière d'occupation du sol et de caractéristiques des sols sont difficiles à acquérir; elles nécessitent une exploration in situ accompagnée d'une prise d'échantillons. Le débit écologique devra néanmoins être accompagné de lâchers à débits plus importants pour permettre le décolmatage. Toutefois, cette modélisation ne pourrait être réalisée que si ces données indispensables sont disponibles et de qualité suffisante.

\section{Bibliographie}

Abdalla O.A.E. \& Al-Rawahi A.S., 2013. Groundwater recharge dams in arid areas as tools for aquifer replenishment and mitigating seawater intrusion: exemple of AlKod, Oman. Environ. Earth Sci., 69, 19511962.

Abedini M.J., Nasseri M. \& Ansari A., 2008. Cluster-based ordinary kriging of piezometric head in West Texas/New Mexico - Testing of hypothesis. J. Hydrol., 351(3-4), 360-367.

Achite M. \& Meddi M., 2004. Estimation du transport solide dans le bassin versant de l'oued Haddad (NordOuest algérien). Sécheresse, 15(4), 367-373.

Achite M. \& Meddi M., 2005. Variabilité spatio-temporelle des apports liquide et solide en zone semi-aride. Cas du bassin versant de l'oued Mina (nord-ouest algérien). Rev. Sci.Eau, 18(special), 37-56.

Achite M.\& Ouillon S., 2007. Suspended sediment transport in semiarid watershed, Wadi abd, Algeria (1973-1995). J. Hydrol., 343, 187-202.

Bergaoui M., Camus H. \& Nouvelot J.F., 1998. Essai de modélisation du transport solide sur les micros bassins versants de Tebaga (Tunisie centrale). Sécheresse, 9(1), 51-57.

Bouanani A., 2004. Hydrologie, transport solide et modélisation. Étude de quelques sous-bassins de la Tafna. Thèse de doctorat: Université de Tlemcen (Algérie).

Bouckaert R.R., 2010. WEKA Manual for Version 3-6-4. Hamilton, New Zealand: University of Waikato.

Bourouba M., 1996. Essai de quantification de l'érosion actuelle à partir des mesures de turbidité en Algérie orientale. Bull. ORSTOM, 16, 232-250.

Bourouba M., 1997. Les variations de la turbidité et leurs relations avec les précipitations et les débits des oueds semi-arides de l'Algérie orientale. Bull. ORSTOM, 17, 345-360.

Bourouba M., 1998. Phénomène de transport solide dans les hauts plateaux orientaux. Cas de l'oued Lougmence et oued Leham dans le bassin de la Hodna. Rev. Sci. Technol., 9, 5-11.

Clarke R.T., 2009. Bias and variance of estimators of suspended sediment load. Hydrol. Sci. J., 35(3-6), 253-261.

Degallier R., 1969. Interprétation des variations naturelles du niveau des nappes. Bull. BRGM, 3(2), 7-56.

Elahcene O. et al., 2013.Étude de la dynamique sédimentaire dans le bassin versant de l'Oued Bellah (Algérie). Hydrol. Sci. J., 58(1), 224-236.

Fareh A., 2006. Approche méthodologique de la cartographie de la sensibilité des sols à l'érosion. Cas du bassin versant de l'Oued El Hachem Wilaya de Tipaza. Mémoire d'ingénieur: Institut National Agronomique, Alger (Algérie).

Ghenim A., 2008. Étude des écoulements et des transports solides dans les régions semi-arides méditerranéennes. Thèse de doctorat : Université de Tlemcen (Algérie) .

Heusch B. \& Millies-Lacroix A., 1971. Une méthode pour estimer l'écoulement et l'érosion dans un bassin. Application au Maghreb. Mines Géol. Rabat, 33, 21-39.

Horowitz A.J., 2002. The use of rating (transport) curves to predict suspended sediment concentration: a matter of temporal resolution. In: Proceedings of the workshop, Turbidity and other sediment surrogates, April 30-May 2, 2002, Reno, NV, USA.

Keddar A., 2011. Impact des aménagements hydrauliques sur le transport solide dans une région semi-aride: cas du bassin des Isser (Algérie). Berlin: Éditions Universitaires Européennes.

Khanchoul K., Jansson M.B.\& Lange Y., 2007. Comparison of suspended sediment yield in two catchments, northeast, Algeria. Z. Geomorphol., 51(1), 63-94. 
Khanchoul K., Boukhrissa Z.E.A., Acidi A. \& Altschul R., 2012. Estimation of suspended sediment transport in the Kebir drainage basin, Algeria. Quat. Int., 262, 25-31.

Lahlou A., 1988. Étude actualisée de l'envasement des barrages au Maroc. Rev. Sci.Eau, 6(3), 337-356.

Li H., Lee J.H. \& Cai M., 2003. Nutrient load estimation methods for rivers. Int. J. Sediment Res., 18(4), 346-351.

Maamar H., 2004. Annuaire hydrogéologique de la nappe alluviale de l'oued El Hachem. Blida, Algérie : Agence Nationale des Ressources Hydrauliques (ANRH).

Meddi M., 1999. Étude de transport solide dans le bassin versant de l'Oued Ebdda (Algérie). Z. Geomorphol., 43, 167-183.

Medejerab A. \& Henia L., 2011. Variations spatiotemporelles de la sécheresse climatique en Algérie nordoccidentale. Courrier du Savoir, 11, 71-79.

Megnounif A., Terfous A. \& Bouanani A., 2003. Production et transport des matières solides en suspension dans le bassin versant de la Haute-Tafna (Nord-Ouest algérien). Rev. Sci. Eau, 16(3), 369-380.

Megnounif A., Terfous A. \& Ouillon S., 2013. A graphical method to study suspended sediment dynamics during flood events in the Wadi Sebdou, NW Algeria (19732004). J. Hydrol., 497, 24-36.

Mimikou M., 1982. An investigation of suspended sediment rating curves in western and northern Greece. Hydrol. Sci. J., 35(3-6), 369-383.

Moatar F. et al., 2007. Évaluation des flux de MES à partir des suivis discrets : méthodes de calcul et incertitudes. In : Conférence "Transports solides et gestion des sédiments en millieux naturels et urbains”, Hôtel de la Communauté urbaine, 28-29 novembre 2007, Lyon, France.

Prathapar S.A. \& Bawain A.A., 2014. Impact of sedimentation on groundwater recharge at Sahalanowt Dam, Salalah, Oman. Water Int., 39(3), 381-393.

Probst J.L. \& Amiotte Suchet P., 1992. Fluvial suspended sediment transport and mechanical erosion in the Maghreb (North Africa). Hydrol. Sci. J., 37, 621-637.

Remini B.\& Hallouche W., 2005. Prévision de l'envasement dans les barrages du Maghreb. Larhyss J., 4, 69-80.

Sogreah, 1967. Étude générale des aires d'irrigation et d'assainissement agricole en Algérie. Dossier. Alger : Ministère de l'Agriculture et de la Réforme agraire.
Szczypta C., 2012. Hydrologie spatiale pour le suivi des sécheresses du bassin méditerranéen. Thèse de doctorat : Institut National Polytechnique de Toulouse (France).

Tadrist N., 2004. Le transport solide de l'Oued El Hachem et l'impact des lâchers sur l'hydraulique de l'Oued. Mémoire de magister : Université Sâad Dahlab de Blida (Algérie).

Terfous A., Megnoufi A. \& Bouanani A., 2001. Étude du transport solide en suspension dans l'oued Mouilah (Nord-Ouest Algérien). Rev. Sci. Eau, 14(2), 173-185.

Touaïbia B., 2000. Érosion-Transport solide-Envasement des barrages. Cas du bassin versant de Mina. Thèse de doctorat d'État: Institut National Agronomique, Alger (Algérie).

Touaibia B., 2010. Problématique de l'érosion et du transport solide en Algérie septentrionale. Sécheresse, 21(4), 333-335.

Ungemach P., 1969. Exploitation des tarissements des nappes d'eaux souterraines. Application à la recherche du coefficient d'emmagasinement en nappe libre, de l'infiltration efficace et de la diffusivité hydraulique. Journées H. Schoeller, Congrès national d'Hydrogéologie, Bordeaux. Mém. B.R.G.M., 76, 271295.

Walling D.E., 1984. The sediment yields of Africa Rivers. In: Walling D.E., Foster S.S.D. \& Wurzel P., eds. Proceedings of the Symposium, Challenges in African hydrology and water resources, July 1984, Harare, Zimbabwe. Wallingford, UK: IAHS Publ. 144, 265-283.

Walling D.E. \& Webb B.W., 1981. The reliability of suspended sediment load data. In: Proceedings of the Symposium, Erosion and sediment transport measurement, June 1981, Florence, Italy. Wallingford, UK: IAHS Publ. 133, 177-194.

Yahiaoui A., 2012. Inondations torrentielles. Cartographie des zones vulnérables en Algérie du Nord (cas de l'oued Mekerra, Wilaya de Sidi Bel Abbès). Thèse de doctorat : École Nationale Polytechnique El Harrach, Alger (Algérie).

Yang G. et al., 2006. Sediment rating parameters and their implications: Yangtze River, China. Geomorphology, 85(3-4), 166-175.

(44 réf.) 